\title{
Thrombolyse 1992, nouvelles stratégies : réalités et rêves
}

Différentes stratégies sont envisagées pour améliorer les traitements thrombolytiques : la synthèse par génie génétique d'activateurs du plasminogène ou $d$ 'autres thrombolytiques plus actifs, plus spécifiques de la fibrine et plus stables; le ciblage de ces agents vers les caillots de fibrine riches en plaquettes, éventuellement par couplage à des anticorps spécifiques; l'inhibition de la thrombogenèse associée à la thrombolyse, notamment en neutralisant la thrombine liée au caillot en voie de dissolution; la modification de la structure des caillots afin de les rendre plus accessibles aux thrombolytiques; enfin, un rêve, qui est en train de se concrétiser, est de rendre la paroi exposée au flux, non thrombogénique. Les techniques modernes de génie génétique permettent d'avancer dans plusieurs de ces voies et certains des produits ou méthodes ainsi créés sont d'ores et déjà en cours d'étude chez l'animal ou d'essais cliniques.

\section{$\mathrm{He} \mathrm{Lu}$ \\ Jeannette Soria Claudine Soria Jacques Caen}

\section{ADRESSES}

H. Lu : maître de conférences associè à la faculté de médecine et pharmacie de Rouen, chercheur à l'Institut des Vaisseaux et du Sang et à l'Inserm U. 353, Paris. J. Soria, praticien hospitalier, Geffacs, laboratoire Sainte-Marie, HôtelDieu, Paris. C. Soria : professeur à la faculté de médecine et pharmacie à Rouen, hôpital Lariboisic̀re et Inserm U. 353, Paris. J. Caen, professeur à la faculté de médecine, université ParisVII, directeur de l'Institut des Vaisseaux et du Sang. IVS, hôpital Lariboisière, 8, rue Guy-

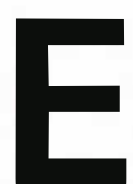

n 1958, le premier agent thrombolytique disponible, la streptokinase (SK), fut utilisé dans le traitement de l'infarctus aigu du myocarde [1]. Pendant les trente ans qui suivirent, grâce aux connaissances accrues du système fibrinolytique $[2$, 3 ], les deux activateurs du plasminogène physiologiques que sont le $\mathrm{t}-\mathrm{PA}$ (activateur tissulaire du plasminogène) et le Scu-PA (pro-urokinase) furent purifiés et leurs ADNc clonés, ce qui permit de les préparer en grande quantité. Actuellement, on dispose de cinq agents thrombolytiques [4] dont les caractéristiques sont résumées dans le Tableau I. La SK et l'urokinase sont les agents de première génération le $\mathrm{t}-\mathrm{PA}$, le scu-PA et l'Apsac (complexe streptokinaseplasminogène dont le centre actif, temporairement bloqué par acylation, est activé au niveau du thrombus par une désacylation spontanée), repré- sentent les agents de deuxième génération. Le principal progrès apporté par ces agents de deuxième génération est d'induire une activation du plasminogène en plasmine au niveau du caillot ; ils évitent donc la dégradation massive du fibrinogène plasmatique. Grâce aux très nombreuses études cliniques résumées récemment $[5,6]$, il apparaît que les traitements thrombolytiques apportent un bénéfice évident dans le traitement de l'infarctus aigu du myocarde : environ $80 \%$ des vaisseaux obturés par des thrombi frais sont recanalisés par l'agent thrombolytique par rapport à $20 \%$ chez les malades traités par un protocole conventionnel. En outre, la réduction de mortalité par le traitement thrombolytique a été confirmé dans tous les essais contrôlés quel que soit le produit utilisé, à condition que le médicament soit administré dans les six heures qui suivent le début des symptômes. Toutefois, les résultats 
cliniques ne sont pas encore totalement satisfaisants : il reste environ $20 \%$ des cas qui ne sont pas sensibles aux thérapeutiques actuellement utilisées, $20 \%$ de réocclusion après traitement thrombolytique et $20 \%$ d'hémorragies et surtout 0,4 à $0,6 \%$ des malades traités ont des hémorragies intracrâniennes. L'étude ISIS-3 (international study in infarct survival) [7], portant sur 41299 patients atteints d'infarctus du myocarde, a montré que le bénéfice induit par les agents de $2^{e}$ génération (rt-PA et Apsac) n'était en fait pas supérieur à celui apporté par la SK. En outre, le risque d'hémorragie cérébrale apparaît légèrement plus élevé avec le rt-PA associé à l'héparine qu'avec la SK. Cela montre que ce n'est pas la chute du fibrinogène qui est en cause dans le risque hémorragique, mais la dégradation des caillots d'hémostase. Selon qu'elles sont récentes ou anciennes, les thromboses veineuses répondent plus ou moins bien aux traitements thrombolytiques, mais l'effet préventif à long terme du syndrome postphlébitique n'a pas encore fait ses preuves. Les traitements thrombolytiques sont, en revanche, très utilisés dans les cas d'embolie pulmonaire sévère, généralement sensible à la thrombolyse.

Pour éviter les échecs, de nouvelles stratégies (figure 1, p. 691) sont actuellement en cours d'investigation [8], les recherches étant dirigées dans trois directions.

- Frapper vite et fort, car une véritable course contre la montre s'installe entre le moment où la thrombose se forme et celui où la thrombolyse est complète, afin de limiter la nécrose fatale. Pour cela, il faut des produits faciles à administrer et qui induisent le plus rapidement possible une forte concentration de plasmine dans les vaisseaux obstrués.

- Limiter la réocclusion. Un effort important est actuellement mis en œuvre pour chercher les produits qui sont les plus efficaces pour bloquer l'activation de la coagulation et l'activation plaquettaire. En outre, il faut limiter la thrombogénicité de la surface qui se trouve exposée à la suite de la dégradation du caillot.

- Enfin le thrombus vieillit, s'organise et devient résistant à la thrombolyse. La stratégie logique serait $\mathrm{m} / \mathrm{s} n^{\circ} 7$, vol. 8 , septembre 92

Tableau I

CARACTÉRISTIQUES DES PRINCIPAUX AGENTS THROMBOLYTIQUES (D'après [5])

\begin{tabular}{|c|c|c|c|c|c|}
\hline & \multicolumn{2}{|c|}{ Première génération } & \multicolumn{3}{|c|}{ Deuxième génération } \\
\hline & SK & UK & rt-PA & scu-PA & Apsac \\
\hline Demi-vie (min) & 23 & 16 & 8 & 7 & 90 \\
\hline Potentialisation par la & & & & & \\
\hline fibrine & $1+$ & $2+$ & $4+$ & $4+$ & $1+$ \\
\hline Durée de & & & & & \\
\hline l'administration & $1 \mathrm{~h}$ & $5-15 \mathrm{~min}$ & $3 \mathrm{~h}$ & $2-3 h$ & $5-10 \mathrm{~min}$ \\
\hline Reperfusion $(\%)$ & $60-70$ & $60-70$ & $60-70$ & $60-70$ & $60-70$ \\
\hline Fibrinogénolyse & $4+$ & $4+$ & $4+$ & $4+$ & $4+$ \\
\hline Temps de reperfusion & & & & & \\
\hline$(\min )$ & 45 & 45 & 45 & 45 & 45 \\
\hline Réocclusion $(\%)$ & 15 & 10 & 20 & ND & 10 \\
\hline Hémorragie $(\%)$ & $4+$ & $4+$ & $4+$ & $4+$ & $4+$ \\
\hline Allergie & Oui & Non & Non & Non & Oui \\
\hline
\end{tabular}

SK : streptokinase ; UK : urokinase ; rt-PA : t-PA recombinant ; scu-PA : pro-urokinase ; Apsac : complexe plasminogène/streptokinase activé par désacylation au niveau du thrombus. L'expression en + représente : pour $1+$ un phénomène d'intensité discrète, $2+$ un phénomène d'intensité modérée, $3+$ un phénomène d'intensité importante et $4+$ un phénomène d'intensité très importante.

donc de modifier la structure du thrombus pour le rendre plus accessible aux enzymes fibrinolytiques, et de prévenir le vieillissement du caillot en évitant la colonisation du thrombus par des cellules qui modifient sa structure.

Ces trois voies de recherche sur lesquelles nous allons faire le point sont illustrées sur la figure 1.

\section{Amélioration des agents thrombolytiques}

Différentes stratégies ont été utilisées (figure 1A).

Obtention de mutants des activateurs du plasminogène par mutagenèse dirigée afin d'améliorer leurs propriétés

- Augmentation de la demi-vie par mutation des sites de glycosylation ou du domaine EGF du t-PA impliqués dans le phénomène de clairance. De nombreuses expériences ont montré que la demi-vie des molécules ainsi modifiées peut être neuf fois plus longue que celle de la molécule mère naturelle. Malheureusement, le bénéfice de tels mutants peut être contrebalancé par une spécificité moins importante pour la fibrine [8].

- Obtention de mutants moins sensibles à l'action des inhibiteurs comme le PAI-1 (plasminogen activator inhibitor) [9]. A première vue, il ne paraît pas que l'obtention de tels mutants puisse apporter un bénéfice important dans les thérapeutiques thrombolytiques car les quantités importantes de t-PA administrées dépassent largement la concentration plasmatique du PAI-1. Toutefois, étant donné la forte quantité de PAI-1 libérée par les plaquettes dans un caillot riche en plaquettes, ces mutants pourraient néanmoins se montrer plus efficaces que les molécules naturelles.

- Obtention de pro-urokinases mutées non transformables en urokines en substituant la Lys 158 par de l'Ala au niveau du site de clivage par la plasmine, dans le but d'améliorer la spécificité d'action sur la fibrine. Ces mutants ont été abandonnés car ils sont cinq fois moins actifs que la molécule mère [10].

Obtention d'hybrides entre le t-PA et la pro-urokinase afin d'apporter simultanément les structures des deux molécules impliquées dans leur activité biologique

L'utilisation d'un hybride composé de la fraction active de la pro-urokinase (molécule amputée de la fraction $\mathrm{N}$ terminale) et de la fraction impliquée dans la fixation du t-PA à la fibrine (Kringles) [11] a donné des résultats décevants : l'affinité pour la fibrine est inférieure à celle du $\mathrm{t}-\mathrm{PA}$, ce qui montre que la géométrie des molécules physiologiques a été très bien 


\section{RÉFÉRENCES}

1. Fletcher AP, Alkjaersig N, Smyrniotis FE, Sherry S. The treatment of patients suffering from early myocardial infarction with massive and prolonged streptokinase therapy. Trans Assoc Am Phys 1958; 71 : 289-96.

2. Caen J, Tobelem G, Soria C. Fibrinolyse et thrombolyse. Paris : Masson, 1986

3. Bachmann F. Fibrinolysis. In : Verstracte M, Vermuylen G, Lynen HR, Arnoult J, eds. Thrombosis and Haemostasis. Leuven : Leuven University Press, 1987, 227-65.

4. Topol EJ. Thrombolytic intervention. In Topol EJ, ed. Textbook of Interventional Cardiology. Philadelphie : W.B. Saunders Company 1990 : 76-121.

5. Marder VJ, Sherry S. Thrombolytic therapy, current status. $N$ Engl Med 1988 ; 318 $1512-20 ; 1585-95$.

6. Arnold AER, Ferdinand RF, Soward AL, Schmidt WG, Lubsen J. Thrombolysis in suspected acute myocardial infarction. Francfort : Universimed Verlag GmbH press, 1990.

7. ISIS-3 collaborative group. ISIS-3 : a randomised comparison of streptokinase vs. tissue plasminogen activator $v s$. anistreplase and of aspirin plus heparin $v s$. aspirin alone among 41299 cases of suspected acute myocardial infarction. Lancet $1992 ; 339$ : 753-70.

8. Lijnen HR, Collen D. Towards the development of improved thrombolytic agents. $\mathrm{Br}$ $J$ Haemotol 1991; $77: 261-6$.

9. Madison EI, Goldsmith EJ, Gerard RD, Gething MJH, Sambrook JF. Serpin-resistant mutants of human tissue plasminogen activator. Nature 1989 ; 339 : 721-4.

10. Collen D, Mao J, Stassen JM, et al. Thrombolytic properties of Lys 158 mutants of recombinant single chain urokinase type plasminogen activator (Scu-PA) in rabbits with jugular vein thrombosis. I Vase Med Biol 1989 ; 1: 46-9.

11. Lijnen HR, Nelles L, Van Hoef B, Demarsin E, Collen D. Characterization of a chimeric plasminogen activator consisting of amino acids $1-274$ of tissue plasminogen activator and amino acids 138-411 of single-chain urokinase-type plasminogen activator. $J$ Biol Chem 1988; 263 : 19083-91.

12. Bode C, Matsueda GR, Hui KY, Haber E. Antibody-directed urokinase : a specific fibrinolytic agent. Science $1985 ; 229$ : 765-7.

13. Runge MS, Quertermous T, Zavodny PJ, et al. A recombinant chimeric plasminogen activator with high affinity for fibrin has increased thrombolytic potency in vitro and in vivo. Proc Natl Acad Sci USA 1991; 88 : 10337-41.

14. Kurokawa T, Iwasa S, Kakinuma A, Stassen JM, Lijnen HR, Collen D. Enhancement of clot lysis in vitro and in vivo with a biospecific monoclonal antibody directed against urokinase-type plasminogen activator. Thromb Haemost 1991 ; 66 : 684-93.
15. Gold HK, Coller BS, Yasuda T, et al. Rapid and sustained coronary artery recanalization with combined bolus injection of tissue plasminogen activator and monoclonal antipla telet GP IIb/IIIa antibody in a canine preparation. Circulation $1988 ; 77: 670-7$.

16. Jang IK, Gold KH, Ziskind AA, et al. Differential sensibility of erythrocyte-rich and platelet rich arterial thrombi to lysis with recom binant tissue type plasminogen activator. A possible explanation for resistance to coronary thrombolysis. Circulation 1989 ; 70 : 920-8.

17. Dewerchin M, Lijnen HR, Stassen JM, al. Effect of chemical conjugation of recombinant single-chain urokinase-type plasminogen activator with monoclonal anti-platelet antibodies, on platelet aggregation and on plasma clo lysis in vitro and in vivo. Blood 1991; 78 1005-18.

18. Bode C, Meinhardt G, Runge MS, et al Platelet-targeted fibrinolysis enhances clot lysis and inhibits platelet aggregation. Circulation $1991 ; 84$ : 805-13.

19. Gardell SJ, Duong LT, Dichlre, et al. Iso lation, characterization, and cloning of a vampire bat salivary plasminogen activator. $J$ Biol Chem 1989 ; 264 : 17947-52.

20. Shebuski RJ, Fujita T, Ramjit DR, et al. Thrombolytic efficacy of IV bolus vampire ba salivary plasminogen activator (bPA) in a rabbit model of femoral arterial thrombosis : comparison to tissue-type plasminogen activator (t PA). Fibrinolysis 1990 ; 4 (suppl 3) : 97 (abstr)

21. Mirshahi M, Soria J, Soria C, et al. Eva luation of the inhibition by heparin and hirudin of coagulation activation during r-t-PAinduced thrombolysis. Blood $1989 ; 74$ 1025-30.

22. Agnelli G, Pascucci C, Cosmi B, Nenci GG. Effects of hirudin and heparin on the binding of new fibrin to the thrombus in t-PA treated rabbit. Thromb Haemost 1991; 66 : $592-7$

23. Märki WE, Wiallis RB. The anticoagulan and antithrombotic properties of hirudins. Thromb Haemost 1990 ; 64 : 344-8.

24. Haskel EJ, Prager NA, Sobel BE, Abendschein DR. Relative efficacy of anti-thrombin compared with antiplatelet agents in accelerating coronary thrombolysis and preventing early reocclusion. Circulation 1991; 83 : 1048-56.

25. Mirshahi MC, Soria J, Neuhart E, et al. Effect of heparin and cnoxaparin on platele interaction with fibrin clots. Thromb Res 1992 ; 65 : 187-91.
" conçue " au cours de l'évolution et qu'il est difficile d'y apporter des améliorations notables.

Complexes permettant de diriger les activateurs du plasminogène sur le thrombus selon le processus de " ciblage"

L'acheminement des activateurs du plasminogène vers le thrombus peut être amélioré par la construction de chimères associant un activateur du plasminogène avec, soit un anticorps antifibrine, soit un anticorps dirigé contre les plaquettes activées qui se lient avec très grande affinité au caillot de fibrine. Plus récemment, des anticorps à double spécificité, antifibrine et anti-plaquettes activées, ont été produits. Ces chimères sont obtenues par des méthodes chimiques ou génétiques. Elles varient en fonction de l'activateur du plasminogène ( $t-P A$ ou pro-urokinase) et du type d'anticorps utilisé (Tableau II). La clé du problème est de choisir des anticorps à forte affinité. Concernant l'anticorps anti-fibrine, il doit reconnaître un épitope non présent sur le fibrinogène, facilement accessible sur le thrombus et de plus présent en quantité suffisante pour que le ciblage soit efficace. En outre, l'épitope reconnu par l'anticorps ne doit pas être libéré du caillot plus vite que le reste de la molécule de fibrine. Pour l'instant, deux types d'anticorps spécifiques de la fibrine ont été utilisés. Le premier est dirigé contre la fraction $\mathrm{N}$ terminale de la chaîne $\beta$ de la fibrine (B $\beta$ 15-42) et le deuxième contre les D-dimères de la fibrine [12-14]. L'anticorps dirigé contre l'extrémité de la chaîne $\beta$ de la fibrine présente une efficacité importante probablement liée à son double rôle : il " cible " l'activateur du plasminogène vers le caillot et, de plus, en se liant au site de polymérisation $\mathrm{B}$ de la fibrine, il gêne la polymérisation de la fibrine et donc l'extension de la thrombose.

Les anticorps anti-plaquettes utilisés furent d'abord dirigés contre des glycoprotéines de la membrane plaquettaire (GP IIb/IIIa) [15] et furent ensuite améliorés par l'utilisation d'anticorps reconnaissant les plaquettes activées. La stratégie consiste à accélérer la lyse des caillots riches en plaquettes, qui sont particulièrement résistants à la thrombolyse [16]. Les $m / s n^{\circ} 7$, vol. 8 , septembre 92 


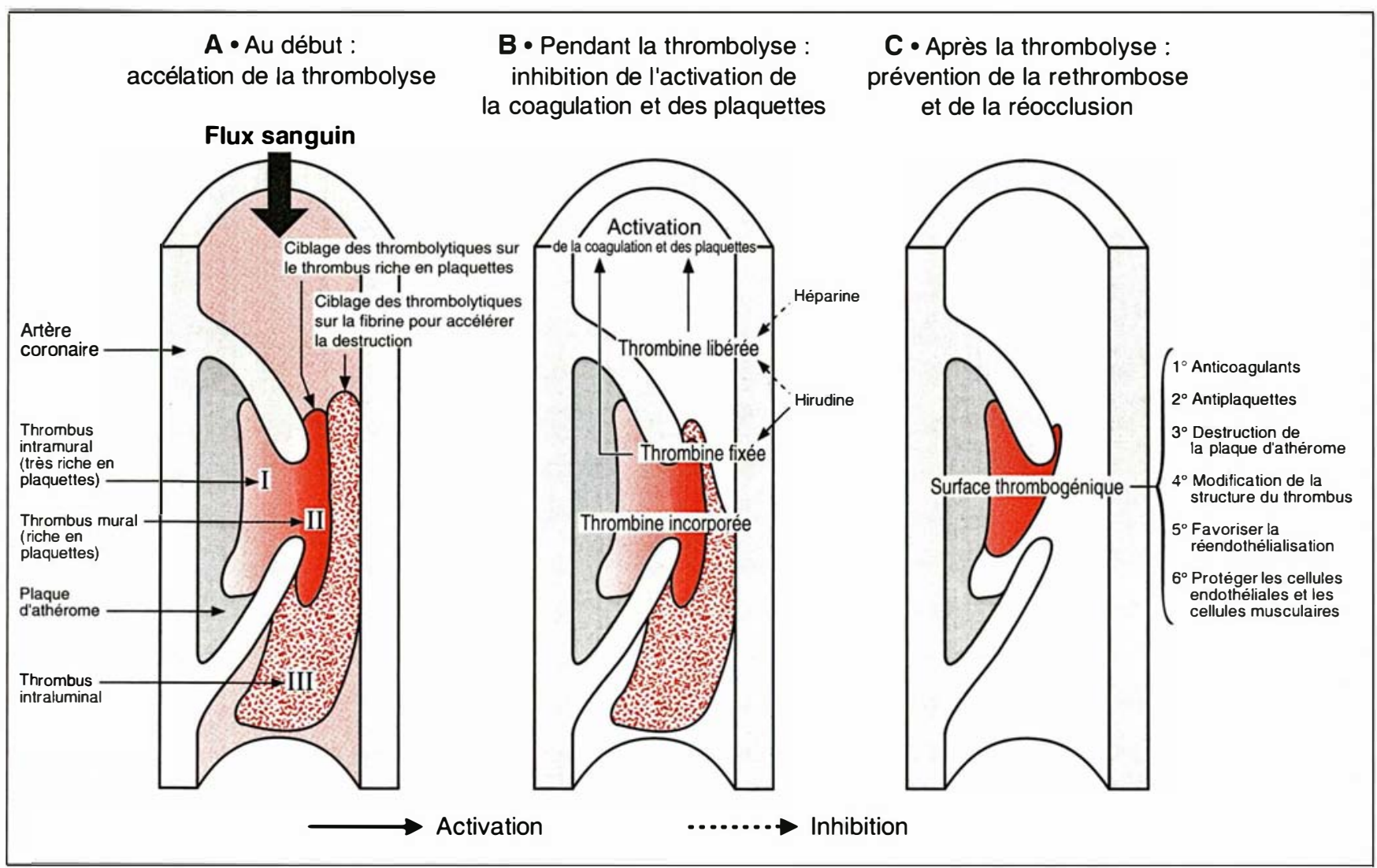

Figure 1. Stratégies pour améliorer les traitements thrombolytiques.

Tableau II

RÉSULTATS DES ESSAIS SUR LES CONDITIONS ENTRE ACTIVATEURS DU PLASMINOGĖNE ET ANTICORPS MONOCLONAL ANTIFIBRINE PERMETTANT LE CIBLAGE DES THROMBOLYTIQUES SUR UN THROMBUS EXPÉRIMENTAL*

\begin{tabular}{|c|c|c|c|c|c|c|}
\hline $\begin{array}{l}\text { Activateurs du } \\
\text { plasminogène }\end{array}$ & $\begin{array}{l}\text { Cible de } \\
\text { l'anticorps }\end{array}$ & $\begin{array}{l}\text { Désignation } \\
\text { des chimères }\end{array}$ & $\begin{array}{l}\text { Animal } \\
\text { d'expérience }\end{array}$ & $\begin{array}{l}\text { L'efficacité de } \\
\text { la thrombolyse } \\
\text { (x fois })^{* *}\end{array}$ & $\begin{array}{l}\text { Spécificité de } \\
\text { la thrombolyse } \\
\text { (x fois })^{* *}\end{array}$ & $\begin{array}{l}\text { Prolongation } \\
\text { de la clairance } \\
\text { (x fois })^{* *}\end{array}$ \\
\hline$t-P A$ & chaîne $B \beta$ & t-PA-59D8 & Lapin & 2,8 & - & - \\
\hline scu-PA & chaîne $B \beta$ & scu-PA-59D8 & Lapin & 29 & - & - \\
\hline \multirow[t]{2}{*}{ scu-PA } & chaîne D-dimère & scu-PA/MA-15C & Hamster & 73 & 15 & 10 \\
\hline & & & Lapin & 8 & 3 & 4 \\
\hline tcu-PA & D-dimère & tcu-PA/MA-15C5 & Lapin & 6 & 0,5 & 6 \\
\hline ScuPA & D-dimère & $\begin{array}{l}\text { rscu-PA/MA- } \\
\text { FUl-74***}\end{array}$ & Hamster & 17 & 0 & 20 \\
\hline
\end{tabular}

* D'après Lijnen HR, Collen D. Thromb Haemost 1991 ; 66 : 103.

** Facteur de multiplication par rapport à l'activateur du plasminogène non combiné à l'anticorps.

*** un anticorps bispécifique.

t-PA: activateur tissulaire du plasminogène; scu-PA: pro-urokinase; tcu-PA: urokinase (two chains urokinaseplasminogen activator).

$m / s n^{\circ} 7$, vol. 8, septembre 92 


\section{RÉFÉRENCES}

26. Winters KJ, Santoro SA, Miletich JP, Eisenberg PR. Relative importance of thrombin compared with plasmin-mediated platelet activation in response to plasminogen activation with streptokinase. Circulation $1991 ; 84: 1552-60$

27. Lu H, Soria C, Cramer EM, et al. Temperature dependence of plasmin-induced platelet activation and inhibition of human platelet. Blood 1991; 77 : 996-1005

28. Hsia J, Hamilton WP, Klciman $\mathrm{N}$, Robert R, Chaitman BR, Ross AM. A comparison between heparin and low-dose aspirin as adjunctive therapy with tissue plasminogen activator for acute myocardial infarction. $N$ Engl J Med 1990 ; 323 : 1433-7.

29. Yasuda T, Dold HK, Fallon JT, et al. Monoclonal antibody against the platelet glycoprotein IIb/IIIa receptor prevents coronary artery reocclusion after reperfusion with recombinant tissue-type plasminogen activator. J Clin Invest $1988 ; 81: 1284-91$

30. Webb RD, Olson RW, Dotson R, Mathis J, Cohen DS. Beneficial effect of CGS 2265/2, a thromboxane receptor antagonist/thromboxane synthetase inhibitor as a adjunct to streptokinase (SK) therapy in a canine model of coronary thrombosis. Thromb Haemost 1991; 65 : 1734 (abstr).

31. Drouct L, Bal dit Sollier C, Cisse M, et al. The antithrombotic effect of KRDS, a lactotransferrin peptide, compared with RGDS. Nouv Rev Fr Hematol 1990 ; 32 : 59-62.

32. Mirshahi MC, Azzarone B, Soria J, Mirshahi F, Soria C. Role of fibroblast in organization and degradation of a fibrin clot. J Lan Clin Med 1991; 117 : 274-81.

33. Soria J, Soria C, Caen JP. A new type of congenital dysfibrinogenemia with defective fibrin lysis. Dusard syndrome : possible relation to thrombosis. $\mathrm{Br} J$ Haematol 1983 ; 53 : 575-86

34. Mosseson MW. Fibrin polymerization and its regulatory role in the hemostasis. $J$ Lab Clin Med 1990 ; 116 : 8-17.

35. Werns SW. Free radical scavengers and leucocyte inhibitor. In : Topol EJ, ed. Textbook of Interventional Cardiology. Philadelphie : W.B. Saunders company, 1990 : 152-72.

36. Forrester JS. Laser angioplasty : current and future prospects. In : Topol EJ, ed. Textbook of Interventional Cardiology. Philadelphie: W.B. Saunders company, 1990 : 738-47

37. Marzelle J, Combe S, Mirshahi MC, et al. Catheter delivered ultrasound dissolves organized thrombi resistant to tissue type plasminogen activator (t-PA). Circulation 1991 ; 84 (suppl II) : 468 (abstr).

38. Wilson JM, Birinyi LK, Salomon RN, Libby $P$. Callow AD, Mulligan RC. Implantation of vascular grafts lined with genetically modified endothelial cells. Science 1989 ; 244 : 1344-6.

\begin{tabular}{|c|c|c|}
\hline \multicolumn{3}{|c|}{$\begin{array}{l}\text { Tableau III } \\
\text { FET INHIBITEUR SUR LA THROMBINE } \\
\text { RINE ET DE L'HIRUDINE. } \\
\text { FFICACITÉ DE LA THROMBOLYSE }\end{array}$} \\
\hline & Héparine & Hirudine \\
\hline $\begin{array}{l}\text { Inhibition de la thrombine* } \\
\text { libre } \\
\text { liée à la fibrine }\end{array}$ & $\begin{array}{l}80 \% \\
20 \%\end{array}$ & $\begin{array}{l}80 \% \\
75 \%\end{array}$ \\
\hline $\begin{array}{l}\text { Inhibition de l'activation } \\
\text { de la coagulation au cours } \\
\text { de la thrombolyse (in vitro } \\
(0,1 \text { unité antithrombine) }\end{array}$ & $50 \%$ & $94 \%$ \\
\hline $\begin{array}{l}\text { Thrombolyse expérimentale } \\
\text { (chien)** } \\
\text { temps de recanalisation } \\
\% \text { de réocclusion }\end{array}$ & $\begin{array}{c}35 \mathrm{~min} \\
80 \%\end{array}$ & $\begin{array}{c}20 \mathrm{~min} \\
0 \%\end{array}$ \\
\hline
\end{tabular}

* D’après [21]. * D’après [24].

résultats sont encourageants chez l'animal, et cette nouvelle approche semble donc prometteuse [17, 18]. Toutefois, le couplage de l'activateur du plasminogène avec un anticorps monoclonal rend la molécule antigénique. Cet inconvénient pourrait être minimisé par l'utilisation d'anticorps humanisés.

Activateurs du plasminogène des animaux vampires

Certains animaux sont hématophages et possèdent dans leur salive une ou plusieurs enzymes capables de dégrader le sang qu'ils ingèrent pour favoriser l'absorption intestinale. Une enzyme très proche du t-PA a été isolée chez une chauve-souris (Desmodus rotundus) qui fait des ravages dans les troupeaux [19]. Elle est déjà produite par génie génétique. L'activité de cette enzyme est très puissante et son activité est 45000 fois plus forte en présence de fibrine qu'en son absence [20]. Ses propriétés antigéniques posent cependant un problème pour une utilisation en pathologie humaine.

\section{Staphylokinase}

Cette substance, extraite du milieu de culture de staphylocoques, forme un complexe actif avec le plasminogène. C'est un agent thrombolytique puissant et, contrairement à la streptokinase, la staphylokinase dégrade la fibrine sans attaquer le fibrinogène. Les essais en clinique n'ont pas encore été effectués, il est donc prématuré de juger de l'intérêt de cette substance.

\section{Association médicamenteuse pour éviter la rethrombose}

(figure 1B)

Cette stratégie est importante car, paradoxalement, la thrombolyse est thrombogène [21, 22]. De plus, après la thrombolyse, la surface vasculaire lésée est réexposée à la circulation et il faut en limiter la thrombogénicité.

\section{Thrombolyse et anticoagulants}

Des résultats récents montrent que l'héparine n'est pas l'anticoagulant idéal pour limiter l'activation de la coagulation liée à la thrombolyse [21, 22]. Ainsi, avons-nous montré que le caillot est un véritable réservoir à thrombine, qu'à l'état basal cette thrombine est en majorité masquée à l'intérieur du caillot, que seule la fraction accessible à la surface est thrombogène et que celle-ci est peu accessible à l'héparine. Au contraire, elle est neutralisable par l'hirudine, polypeptide isolée de la sangsue et préparé par génie génétique [23] (Tableau III).

Lors de la thrombolyse, le caillot est dégradé progressivement à partir de sa surface et la thrombine qui était enfouie à l'intérieur va être progres- 
sivement démasquée. Cela explique que l'hirudine se soit montrée beaucoup plus efficace que l'héparine dans la neutralisation de l'activation de la coagulation induite par la thrombolyse (Tableau III). Dès lors, l'association d'un agent thrombolytique et d'hirudine est séduisante pour limiter les rethromboses. Cette stratégie s'est déjà montrée efficace chez l'animal [24]. En outre, l'hirudine peut également limiter efficacement l'activation plaquettaire induite par la thrombine car, contrairement à l'héparine, elle inhibe aussi bien la thrombine liée au caillot ou à la matrice extracellulaire qui se trouve exposée. En bref, l'hirudine sans antiplaquettaires prévient totalement la réocclusion tandis que, dans les mêmes conditions, l'héparine est inactive (Tableau III) [24] et exerce même une action délétère en favorisant la rétention des plaquettes sur le caillot de fibrine [25].

Thrombolyse et agents antiplaquettaires

Les plaquettes sont activées au cours de la thrombolyse. Cette activation est vraisemblablement occasionnée par la thrombine libérée par la thrombolyse [26, 27]. L'inhibition de l'activation plaquettaire peut accélérer la recanalisation et réduire la réocclusion. L'aspirine ne prévient que partiellement les réocclusions [28]. D'autres agents antiplaquettaires sont à l'essai actuellement chez l'animal. Les inhibiteurs dirigés contre la GP IIb/IIIa activé (glycoprotéine responsable de l'agrégation des plaquettes entre elles) ont donné des résultats encourageants [29]. Les inhibiteurs des récepteurs et de la synthèse du thromboxane $A_{2}$ seraient également plus actifs que l'aspirine [30].

Les connaissances récentes sur les interactions cellulaires et les interactions plaquettes/sous-endothélium incitent à analyser l'effet de peptides inhibiteurs de cette interaction [31]. Les études sont trop récentes pour qu'on puisse en tirer des conclusions définitives sur la prévention des réocclusions après thrombolyse.

Amélioration de la thrombolyse par des drogues modifiant la structuration du caillot

Pendant et après la thrombolyse, la thrombose se reforme sur le vaisseau lésé. Nous avons montré que la thrombolyse dépendait de la structure du caillot [32] et que certains caillots même frais sont inaccessibles aux enzymes fibrinolytiques [33]. La modification de la polymérisation de la fibrine par des anticorps ou, par des substances chimiques afin de rendre le thrombus en formation plus destructible par la plasmine, est donc également une voie séduisante [34]. Association avec des capteurs de radicaux libres

La revascularisation d'un cœur anoxique par un milieu contenant de l'oxygène s'accompagne de troubles sévères avec contracture et nécrose cellulaire. Ce phénomène a été appelé " paradoxe de l'oxygène ". La confirmation du rôle des radicaux libres dans ce processus a été apportée par la mise en évidence d'une augmentation de peroxides lipidiques et par l'effet protecteur des antioxydants et de capteurs de radicaux libres sur les lésions [35]. L'administration de tels produits au cours des traitements thrombolytiques est actuellement à l'essai.

\section{Dégradation des thrombi par des procédés mécaniques (figure 1C)}

L'avantage de tels procédés (lasers et ultrasons) est la rapidité avec laquelle un vaisseau est désobstrué $[36,37]$. En outre, l'avantage de ces méthodes est de dégrader aussi bien les thrombi frais formés de fibrine que les thrombi âgés organisés. Toutefois, les résultats sont encore fragmentaires car la difficulté réside dans le fait qu'il faut dégrader le caillot sans léser la paroi vasculaire. De nombreux efforts sont actuellement en cours pour améliorer le matériel et la précision de la manœuvre. De ce fait, pour l'instant, ces procédés ne peuvent être réservés qu'aux embolies pulmonaires tellement massives qu'elles seraient mortelles sans une intervention extrêmement rapide.

\section{La prévention du vieillissement du caillot}

C'est un rêve qui concerne à la fois la prévention pré- et post-thrombolytique. Au cours de leur vieillissement les caillots se modifient et deviennent résistants à la thrombolyse, principa- lement du fait de leur envahissement par les fibroblastes [32]. Les fibroblastes inhibent la thrombolyse en sécrétant du collagène qui bloque les sites de la fibrine attaquables par la plasmine [32]. Nous suggérons que l'envahissement du caillot par les fibroblastes et la prolifération de ces cellules résultent de la libération au sein du caillot de cytokines et de facteurs de croissance libérés par les plaquettes et les leucocytes (étude en cours).

\section{Rendre la paroi non thrombogénique}

Ce rêve est en train de se concrétiser. En effet, des cellules endothéliales ont pu être greffées sur un vaisseau lésé dans un modèle animal [38]. De plus, on peut envisager aujourd'hui de greffer des cellules endothéliales génétiquement recombinées, sécrétant de l'activateur du plasminogène et, par conséquent, non thrombogéniques.

\section{Conclusion}

Les thérapeutiques thrombolytiques ont amélioré de façon indiscutable le traitement des maladies thrombotiques et, en particulier, celui de l'infarctus du myocarde. De gros efforts ont été réalisés pour améliorer ces thérapeutiques, mais il reste encore beaucoup à faire. En effet, les traitements thrombolytiques doivent répondre à quatre critères : (1) ils doivent présenter l'efficacité maximale avec un risque hémorragique minimal. Le thrombolytique idéal n'est pas encore trouvé car aucun agent thrombolytique n'est capable de discerner les caillots d'hémostase des thrombi pathologiques; (2) le problème du coût de ces thérapeutiques reste un problème majeur. Le $r$-tPA assure une dégradation très rapide des caillots, mais son prix de revient limite son utilisation, d'autant que les résultats des études cliniques n'ont pas montré une nette amélioration par rapport à la streptokinase. L'association de plusieurs médicaments et la fabrication de chimères peut laisser penser que la posologie pourrait être réduite par rapport aux activateurs du plasminogène natifs car, dans les essais expérimentaux chez l'animal, ils se sont montrés plus actifs et leur durée de vie est prolon- 
gée ; (3) la thrombolyse doit être adaptée à la composition du thrombus. En effet le thrombus n'est pas un simple caillot de fibrine car les plaquettes, comme les cellules sanguines et mésenchymateuses, colonisent le thrombus, qui acquiert ainsi une résistance particulière aux agents thrombolytiques. L'association des médicaments antiplaquettaires aux agents thrombolytiques s'est avérée efficace. Mais pour mieux agir, au niveau du thrombus lui-même, un ciblage des thrombi riches en plaquettes par des anticorps monoclonaux anti-plaquettes activées a fait l'objet d'essais qui se révèlent encourageants chez l'animal d'expérience ; (4) enfin, l'idéal serait non seulement de modifier les agents thrombolytiques eux-mêmes, mais d'agir en amont en rendant les caillots plus sensibles aux substances thrombolytiques, tout en rendant la paroi non thrombogénique

\section{TIRÉS A PART}

\section{Summary}

1992 thrombolysis, new strategies : dreams and reality

Thrombolytic therapy proved effective in treating thrombotic diseases and beneficial to the patients with myocardial infarction. At present, great efforts are being made to induce the lysis of aged thrombi, to reduce reocclusion, rethrombosis rate and bleeding complications that occur during and after this therapy, and finally to improve its long-term benefit. In the cases of acute myocardial infarction, the association of this therapy with anticoagulant agent such as heparin, was proved to accelerate lysis of thrombi and to partially prevent reocclusion. The substitution of heparin by hirudin, a specific and potent thrombin inhibitor is under experimental evaluation. The novel thrombolytic agents prepared by genetic engineering may have an improved thromboly- tic efficiency and a reduced risk of bleeding complications, as suggested by the results in experimental animal models.

The strategies to promote the lysis of the thrombi that are resistant to thrombolytic agents are also emerging : targeting of the thrombolytic agent to activated platelets may accelerate the lysis of platelet-rich thrombi ; modifying fibrin structure and preventing the organization of fibrin clot may reduce the thrombolytic resistance ; finally destroying aged thrombi may be accomplished by mechanical thrombolysis. However, as the impaired vessel wall remains thrombogenic, future work should be directed towards repairing the damaged surfaces in order to prevent reocclusion.

H. Lu.

\section{FLASH}

\section{TEMPÉTE SUR LA GREFFE DE MYOBLASTES DANS LES MYOPATHIES}

La stratégie thérapeutique consistant à greffer des myoblastes nessee pour fonder sa propre société privée (Cell Therapy normaux à des malades souffrant de myopathie de Duchenne a Research Foundation/ à Memphis, amena de nombreux scientifiété présentée dans médecine/sciences [1]. Les bases expérimen- ques et l'administration de la FDA (Food and Drug Administratales et théoriques de cette approche sont : la capacité des myo- tion) à poser des questions sur l'impartialité des données, voire blastes à fusionner avec des myotubes de sujets déficients en même sur le caractère éthique des études menées dans la société dystrophine, permettant ainsi une compensation, au moins par- de Peter Law. Une enquête officielle de la FDA est à l'heure tielle, du déficit chez ces malades; et la très longue durée de actuelle en cours. Plus récemment encore, à l'occasion de la Gorvie supposée des myotubes chimériques ainsi obtenus. Cepen- don Conference sur la Génétique et la Biologie Moléculaire du dant, la greffe de myoblastes allogéniques entraîne une réponse muscle (14 au 19 juin 1992 dans le New Hampshire, USA) un immune qui nécessite l'utilisation d'immunosuppresseur. Après groupe de biologistes cellulaires et moléculaires signa un texte un petit nombre d'études expérimentales chez l'animal, très appelant à un moratoire sur les essais humains de greffes de myomoyennement concluantes, cependant, une demi-douzaine d'équi- blastes. Leur argumentation est basée sur l'absence de tout résulpes débutèrent des essais cliniques chez des enfants atteints de myopathie de Duchenne. Au bout de deux années, le consensus semble presque exister entre ces équipes : il n'existe pas d'argument indiquant une amélioration des symptômes chez les malades traités. Cette conclusion n'est cependant pas acceptée par tous. A un récent congrès organisé à Pittsburgh (PA, USA) Peter Law présentait une série impressionnante de résultats positat prouvé et tangible de ces essais, sur le risque qu'ils font courir aux malades du fait de l'utilisation d'immunosuppresseurs, et sur I'insuffisance des essais chez l'animal. Ces scientifiques, incluant notamment Jean-Pierre Changeux, nouveau président du Comité National Consultatif d'Ethique (France), appellent dans leur texte au développement et à l'approfondissement des modèles expérimentaux [3].

tifs chez 81 \% des 32 garçons myopathes de 6 à 14 ans auxquels avaient été injectés jusqu'à 50 milliards de myoblastes, prélevés chez le père ou chez un frère sain et multipliés par culture ex vivo [2]. La discordance entre ces résultats et ceux de tou- [1. Labrecque C et al. médecine/sciences 1991; 7 : 821-9.] tes les autres équipes, le fait que la série de Peter Law ait été [2. Thompson L. Science 1992; 257: $472-4$. exclusivement obtenue après qu'il eut quitté I'Université du Ten- 13. Thompson L. Science 1992; 257 : 738.$]$ 